\title{
First order superconducting transition near a ferromagnetic quantum critical point
}

\author{
Andrey V. Chubukov' ${ }^{1}$, Alexander M. Finkel'stein ${ }^{2}$, Robert Haslinger ${ }^{3}$, and Dirk K. Morr ${ }^{4}$ \\ 1 Department of Physics, University of Wisconsin, Madison, WI 53706 \\ 2 Department of Physics, Weizmann Institute of Sciences, Rehovot, Israel \\ 3 Los Alamos National Laboratory, Los Alamos, NM 87545 \\ 4 Department of Physics, University of Illinois at Chicago, Chicago IL 60607
}

(Dated: November 4, 2018)

\begin{abstract}
We address the issue of how triplet superconductivity emerges in an electronic system near a ferromagnetic quantum critical point (FQCP). Previous studies found that the superconducting transition is of second order, and $T_{c}$ is strongly reduced near the FQCP due to pair-breaking effects from thermal spin fluctuations. In contrast, we demonstrate that near the FQCP, the system avoids pair-breaking effects by undergoing a first order transition at a much larger $T_{c}$. A second order superconducting transition emerges only at some distance from the FQCP.

PACS numbers: PACS numbers: 74.25.-q
\end{abstract}

Superconductivity near a magnetic instability is a topic of current interest in condensed-matter physics. Magnetically mediated pairing near an antiferromagnetic instability is a candidate scenario for $d$-wave superconductivity in the cuprates and heavy fermions compounds (for a recent review see 11). The emergence of superconductivity, mediated by the exchange of ferromagnetic spin fluctuations, is also expected near ferromagnetic transitions. Ferromagnetic exchange yields Cooper pairs with $S=1$ and therefore generally gives rise to triplet superconductivity. This type of pairing was originally suggested by Anderson and Morel [2] for ${ }^{3} \mathrm{He}$. In recent years, an intensive search has focused on superconductivity in compounds which can be tuned to a ferromagnetic quantum critical point (FQCP) by varying either pressure or chemical composition. Among the studied systems are $\mathrm{MnSi}$, and the heavy fermion compound $\mathrm{UGe}_{2}$ (for an experimental review, see Ref. [3]).

The emergence of superconductivity in electronic systems close to a ferromagnetic instability has recently been studied by three groups, who solved a linearized gap equation within the Eliashberg formalism [4, 5, 6. For both two- (2D) and three-dimensional (3D) systems, their analysis yielded a superconducting transition temperature, $T_{c}^{l}$ ('l' stands for linearized), that substantially decreases as the system approaches criticality, eventually vanishing at the FQCP.

The physical origin of the decrease in $T_{c}^{l}$ near the FQCP lies in the presence of thermal spin fluctuations which behave like magnetic impurities whose scattering potential diverges as the critical point is approached [4, 5, 6. This behavior is reflected in the fermionic self-energy in the normal state, $\Sigma\left(\omega_{n}\right) \propto i T \sum_{m} \operatorname{sign}\left(\omega_{n}\right) \chi_{L}\left(\omega_{m}-\right.$ $\left.\omega_{n}\right)$, where $\chi_{L}(\omega)=\int d^{D-1} q \chi(q, \omega)$ is the "local" spin susceptibility. Since $\chi_{L}(\omega=0)$ diverges at the FQCP for $D \leq 3$ (assuming $\chi(q, 0)=\chi_{0} /\left(\xi^{-2}+q^{2}\right)$ with $\xi \rightarrow \infty)$, the dominant contribution to $\Sigma\left(\omega_{n}\right)$ comes from the $n=m$ term in the frequency sum, i.e., from classical, thermal spin fluctuations. These fluctuations scatter with a finite momentum and zero frequency transfer; hence their similarity with magnetic impurities. This leads to $\Sigma(\omega)=i \gamma \operatorname{sign} \omega$ with $\gamma \propto T \xi^{3-D}$ for system dimension $D<3$ and $\gamma \propto T \log \xi$ for $D=3$.

The analogy with magnetic impurities extends to the pairing problem in which thermal spin fluctuations close to the FQCP tend to break Cooper pairs and hence lower the temperature of the superconducting transition[7. This strong pair breaking effect is reflected in the gap equation where the divergent contributions to the selfenergy $\Sigma(\omega)$ and to the pairing vertex are not cancelled out in the equation for the pairing gap $\Delta(\omega)$ [8] (the ratio of divergent terms is 3 to 1 in our case). Simple estimates show that the linearized gap equation does not have a solution above $\gamma \sim T_{c 0}$ where $T_{c 0}$ is the transition temperature in the absence of thermal fluctuations. Hence, when the FQCP is approached, $T_{c}^{l}$ vanishes as $T_{c}^{l} \propto \xi^{D-3}$ in $D<3$, and $T_{c}^{l} \propto 1 / \log \xi$ for $D=3$. Numerical solutions of the linearized Eliashberg equations near a ferromagnetic instability demonstrate precisely this kind of behavior - $T_{c}^{l}$ falls off when the FQCP is approached more rapidly in $2 D$ [4 than in $3 D$ [5, 6] (Refs. [5, 6] obtained a small finite $T_{c}^{l}$ at criticality by using a selfconsistent approach that goes beyond Eliashberg theory for the self-energy, but still neglects vertex corrections).

In this letter, we argue that the actual behavior of the system is different from that discussed in Refs. [4, 5, 6. Specifically, we show that close to a FQCP, superconductivity emerges via a first order phase transition at $T_{c} \sim T_{c 0}$. The much smaller $T_{c}^{l}$ previously obtained by solving the linearized gap equation is just the end point of the temperature hysteresis loop, at which the normal state becomes unstable. The first indication that the pairing problem near the FQCP is unconventional comes from the observation that at $T=0$, dangerous thermal fluctuations are absent, and hence the pairing gap $\Delta(\omega)$ should generally be of the order of $T_{c 0}$. Explicit calculations confirm this (see below). The second indication is that in the presence of a large gap, the spectrum of ferromagnetic spin fluctuations changes due to feedback effects from the pairing. For the ABM phase of triplet superconductivity, which we consider in the following, this feedback is different for $\chi_{z z}$ and $\chi_{ \pm}$[2] (assuming that the spin of the Cooper pair, $\vec{S}_{c p}$, lies in the xy-plane). At the FQCP, massless excitations survive in $\chi_{ \pm}$, but 
not in $\chi_{z z}$ which in the presence of a pairing gap describes massive longitudinal spin fluctuations. Due to this distinction between $\chi_{ \pm}$and $\chi_{z z}$, the ratio of the divergent terms in the self-energy and the pairing vertex is $\left[2 \chi_{ \pm}^{L}(0)+\chi_{z z}^{L}(0)\right] /\left[2 \chi_{ \pm}^{L}(0)-\chi_{z z}^{L}(0)\right]$. If $\chi_{ \pm}^{L}$ and $\chi_{z z}^{L}$ behaved identically, this ratio would be 3 , and pairbreaking effects of thermal fluctuations would be crucial. When only $\chi_{ \pm}^{L}(0)$ diverges, the above ratio is 1 and the divergent terms from the fermionic self-energy and the pairing vertex cancel out in the gap equation. This in turn implies that the superconducting state with a large gap remains stable well above $T_{c}^{l}$. This behavior, however, cannot extend to small $\Delta(\omega)$ since then $\chi_{z z}^{L}(0)$ cannot be neglected, the above ratio of divergent terms becomes 3 and no cancellation occurs. We therefore expect that at the FQCP, the solution with a finite gap should survive up to the end point at $T_{c}^{n l} \sim T_{c 0} \gg T_{c}^{l}$ ('nl' stands for the solution of the nonlinear equation) where it becomes unstable. In other words, over some range of $T$, both the normal state and the state with a large gap are locally stable. This is a classic scenario for a first order transition. Note, that this behavior is very different from that for singlet pairing, mediated by antiferromagnetic spin fluctuations, where the divergent contributions to $\Sigma(\omega)$ and to the pairing vertex cancel each other even in the linearized gap equation. As a result, the divergence of the thermal self-energy at criticality does not affect $T_{c}^{l}$ [9] which saturates at a finite value for $\xi=\infty$.

In the remainder of this paper we compute $T_{c}^{n l}$ from the full set of nonlinear Eliashberg equations and show that it saturates at a finite value at the FQCP. We will not attempt to compute the actual $T_{c}$ (this would require the analysis of the condensation energy). However, since $T_{c}^{l}$ vanishes at the FQCP and $T_{c}^{n l}$ stays finite, $T_{c}$ near criticality should be comparable to $T_{c}^{n l}$.

Our starting point is the spin-fermion model, which describes the interaction of low-energy fermions with their own spin degrees of freedom, $\mathbf{S}_{\mathbf{q}}$, whose propagator is peaked at $q=0$. The same model was used in earlier studies [4, 6, 6, 9, 10. We assume that $T_{c 0}$ is much less than the Fermi energy $E_{F}$, implying that the pairing instability involves only fermions near the Fermi surface. The model is described by the Hamiltonian

$$
\begin{aligned}
\mathcal{H}= & \sum_{\mathbf{k}, \alpha} \mathbf{v}_{\mathbf{F}}\left(\mathbf{k}-\mathbf{k}_{F}\right) c_{\mathbf{k}, \alpha}^{\dagger} c_{\mathbf{k}, \alpha}+\sum_{q} \chi^{-1}(\mathbf{q}) \mathbf{S}_{\mathbf{q}} \mathbf{S}_{-\mathbf{q}} \\
& +g \sum_{\mathbf{q}, \mathbf{k}, \alpha, \beta} c_{\mathbf{k}+\mathbf{q}, \alpha}^{\dagger} \sigma_{\alpha, \beta} c_{\mathbf{k}, \beta} \cdot \mathbf{S}_{-\mathbf{q}} .
\end{aligned}
$$

where the spin-fermion coupling, $g$, the Fermi velocity, $v_{F}$ (we assume a circular Fermi surface), and the static spin propagator $\chi(q, 0)$ are input parameters. While the upper energy cutoff is in general also an input parameter, our results are cutoff independent for the pairing problem considered here. The dynamical part of $\chi\left(q, \Omega_{m}\right)=\chi_{0} /\left[q^{2}+\xi^{-2}+\Pi\left(q, \Omega_{m}\right)\right]$ arises from the interaction with the low-energy fermions and is explicitly calculated. While we restrict our consideration to $D=2$, our conclusions are also valid for $3 D$ systems.
We assume that the static $\chi(q, 0)$ has the conventional lorentzian form with a weakly temperature dependent $\xi$. This form of $\chi(q, 0)$ was recently questioned [11, 12] since far away from criticality, the static spin susceptibility possesses singular low-energy Fermi liquid corrections that give rise to a universal $|q|$ dependence of $\chi(q, 0)$, and a $T$ dependence of $\xi^{-1}$. It is unclear, however, whether these singular corrections survive in the quantum critical regime, so we restrict with the conventional form of $\chi(q, 0)$ without further justifications.

Near the critical point, a conventional perturbation theory in the spin-fermion coupling (for which $\Sigma=$ $\Pi=0$ is the point of departure) holds in powers of $\lambda=g^{2} \chi_{0} /\left(4 \pi v_{F} \xi^{-1}\right)$, i.e., the quantum-critical region falls into the strong coupling limit. An approach for dealing with a strong coupling problem is the Eliashberg theory [13]. Its validity requires certain conditions to be met. We proceed assuming that the Eliashberg theory is valid, and then discuss what restrictions are necessary.

We first consider the situation right at the FQCP where $\xi^{-1}=0$. In the normal state, the dynamical part of the spin polarization operator, $\Pi\left(q, \Omega_{m}\right)$, is independent of the fermionic self-energy, $\Sigma\left(q, \omega_{n}\right)$ (but not vice versa), as the essential momenta for $\Pi\left(q, \Omega_{m}\right)$ are those with $v_{F} q \gg \Omega_{m}, \Sigma$. As a result, $\Pi\left(q, \Omega_{m}\right)$ has the same form as for free fermions, i.e., for $\left|\Omega_{m}\right| \ll v_{F} q$, $\Pi\left(q, \Omega_{m}\right)=F\left(\Omega_{m}\right) /\left(v_{F} q\right)$ where $F\left(\Omega_{m}\right)=\alpha k_{F}^{2}\left|\Omega_{m}\right|$, $\alpha=g^{2} \chi_{0} /\left(2 \pi E_{F}\right)$, and $E_{F}=k_{F} v_{F} / 2$. At the same time, $\Sigma(\omega)$ is determined by $\Pi$ and given by $\Sigma\left(\omega_{m}\right)=$ $\omega_{0}^{1 / 3} \omega_{m}^{2 / 3}$, where $\omega_{0}=(3 \sqrt{3} / 4) \alpha^{2} E_{F}$. The non-Fermi liquid, $\omega^{2 / 3}$-dependence of the self-energy in $2 \mathrm{D}$ is due to the divergence of the perturbation theory at the FQCP. This form was earlier obtained in Ref. [14].

In the superconducting state, the equations for two components of $F(\Omega)\left(F_{z z}\right.$ and $\left.F_{x x}=F_{y y}\right)$ are coupled to the equation for the pairing gap $\Delta(\omega)$. Similar to the normal state, none of these quantities explicitly depends on $\Sigma$. As a result, one needs to self-consistently solve a set of three coupled equations for the two components of $F$ and $\Delta$. The derivation of the Eliashberg equations is quite straightforward and will not be presented here. With the Cooper pair spin lying in the xy-plane, the coupled equations for $\Delta(\omega), F_{z z}(\Omega)=F_{-}$and $F_{x x}=$ $F_{y y}=F_{+}$at the FQCP have the form

$$
\begin{aligned}
& \Delta\left(\omega_{n}\right)=\frac{4 \pi}{9} \omega_{0}^{1 / 3} T \sum_{m} \frac{1}{\sqrt{\omega_{m}^{2}+\Delta^{2}\left(\omega_{m}\right)}} \\
& \times\left\{\frac{2 \omega_{m}}{\left[F_{+}\left(T, \Delta, \omega_{m}-\omega_{n}\right)\right]^{1 / 3}}\left[\frac{\Delta\left(\omega_{m}\right)}{\omega_{m}}-\frac{\Delta\left(\omega_{n}\right)}{\omega_{n}}\right]\right. \\
& \left.-\frac{\omega_{m}}{\left[F_{-}\left(T, \Delta, \omega_{m}-\omega_{n}\right)\right]^{1 / 3}}\left[\frac{\Delta\left(\omega_{m}\right)}{\omega_{m}}+\frac{\Delta\left(\omega_{n}\right)}{\omega_{n}}\right]\right\}
\end{aligned}
$$

and

$$
\begin{aligned}
& F_{ \pm}\left(T, \Delta, \omega_{m}-\omega_{n}\right)= \\
& \pi T \sum_{n}\left[1-\frac{\omega_{n}\left(\omega_{m}\right) \pm \Delta\left(\omega_{n}\right) \Delta\left(\omega_{m}\right)}{\sqrt{\omega_{n}^{2}+\Delta^{2}\left(\omega_{n}\right)} \sqrt{\omega_{m}^{2}+\Delta^{2}\left(\omega_{m}\right)}}\right]
\end{aligned}
$$


As anticipated, $F_{+}(T, \Delta, 0)=0$ vanishes implying that the corresponding susceptibilities, $\chi_{x x}$ and $\chi_{y y}$, describe massless modes. The vanishing of $F_{+}(T, \Delta, 0)$, however, is not dangerous as it is compensated by the simultaneous vanishing of the numerator in Eq.(2). Exactly the same cancellation of divergences occurs in the gap equation for $d$-wave pairing due to antiferromagnetic spin fluctuations. The vanishing of $F_{-}(T, \Delta, 0)$ would be dangerous, but for finite $\Delta, F_{-}(T, \Delta, 0)=$ $2 \pi T \sum_{n} \Delta^{2}\left(\omega_{n}\right) / \sqrt{\omega_{n}^{2}+\Delta^{2}\left(\omega_{n}\right)}$ is also finite, i.e. the longitudinal spin excitation described by $\chi_{z z}^{-1}(\omega=0) \propto$ $q^{2}+F_{-}(T, \Delta, 0) /\left(v_{F} q\right)$ is massive. In contrast, for the linearized gap equation $\Delta$ is vanishingly small, $F_{-}(T, \Delta, 0)$ vanishes, and the r.h.s of Eq.(2) diverges. Due to this divergence, the linearized gap equation does not have a solution down to $T=0$. Note, that the only energy scale in the Eliashberg equations is $\omega_{0}$, which can be eliminated by rescaling both temperature and the gap in units of $\omega_{0}$. The gap equation is then fully universal, which implies that the mass in $\chi_{z z}$ and the typical momentum $q$ for the pairing problem are both of order $\omega_{0} / v_{F}$.

In Fig. 1 1 a we present the numerical solution for $\Delta(T)$ at the lowest Matsubara frequency, $\omega_{m}=\pi T$. As expected for a first order phase transition, the gap changes discontinuously from a finite value to zero at $T_{c}^{n l} \sim$ $0.015 \omega_{0}$. The inset shows that the discontinuous jump in $\Delta$ occurs for all Matsubara frequencies.

We next study the situation at finite $\xi$ and verify whether the first order superconducting transition becomes second order at some distance from the FQCP. Away from criticality, the equations for $F_{ \pm}\left(T, \Delta, \omega_{m}\right)$ retain their form, but in the gap equation, the factors $F_{ \pm}\left(T, \Delta, \omega_{m}\right)$ are replaced by $F_{ \pm}\left(T, \Delta, \omega_{m}\right) / I^{3}\left(\beta_{ \pm}\right)$, where $I\left(\beta_{ \pm}\right)$by itself depends on $F_{ \pm}\left(T, \Delta, \omega_{m}\right)$ through

$$
I\left(\beta_{ \pm}\right)=\frac{3 \sqrt{3}}{2 \pi} \int_{0}^{\infty} d x \frac{x}{1+\beta_{ \pm} x+x^{3}},
$$

Here $\beta_{ \pm}=b /\left[F_{ \pm}\left(T, \Delta, \Omega_{m}\right) / \omega_{0}\right]^{2 / 3}$, and $b=$ $(8 / 3 \sqrt{3})^{2 / 3}\left(\alpha k_{F} \xi\right)^{-2}$ measures the deviation from the FQCP $(b=0$ at the FQCP). The integral can be expressed in elementary functions. One can easily verify that for finite $\xi$, the gap equation does not contain any divergence, even at infinitesimally small $\Delta$, and hence both $T_{c}^{n l}$ and $T_{c}^{l}$ are nonzero.

In Fig. 1 $1 \mathrm{~b}$ we plot $\Delta(T, i \pi T)$ for $b=2$. We clearly see that the temperature dependence of the gap is now continuous, in marked distinction to Fig. 17a. The inset shows that the continuous evolution of $\Delta$ holds for all Matsubara frequencies. This result implies that for large enough $b>b_{c}$, the transition is of second order. To locate the tricritical point $b=b_{c}$, we plot in Fig. 11c the magnitude of the jump of $\Delta(T, i \pi T)$ at $T_{c}^{n l}$ as a function of $b$. We see that the gap discontinuity disappears at $b_{c} \approx 1.2$. For completeness, in Fig. 11d we plot the zero temperature gap versus $b$. We see that it changes gradually, without a singularity at $b_{c}$.

The phase diagram that emerges from our studies is presented in Fig. 2. The actual superconducting tran-

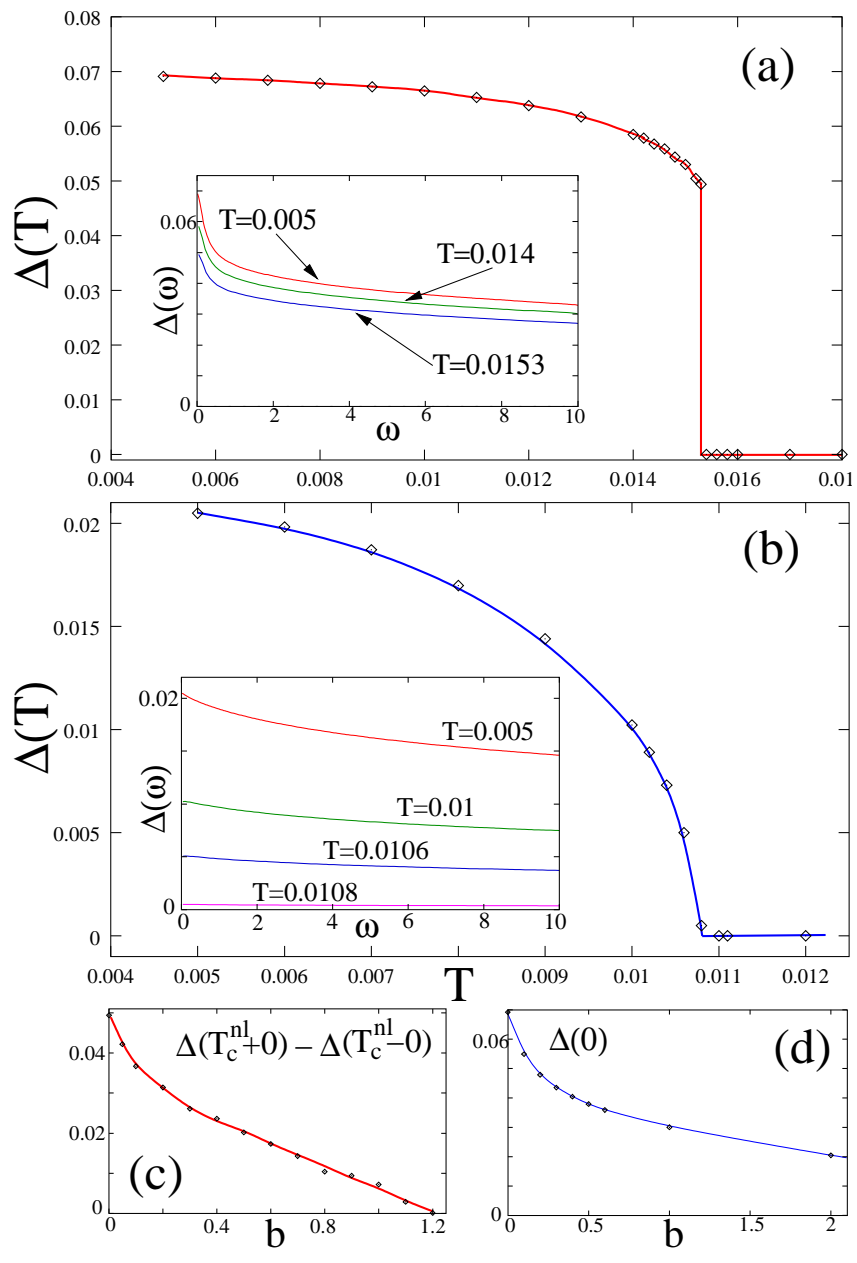

FIG. 1: (a) Temperature dependence of $\Delta\left(T, \omega_{m}\right)$ at the lowest Matsubara frequency $\omega_{m}=\pi T$ for $b=0$. The lines are a guide for the eye. $\Delta, T$ and $\omega_{m}$ are in units of $\omega_{0}$ (see text). The discontinuity of $\Delta(T)$ at 0.015 indicates a first order transition. The inset shows $\Delta(\omega)$ versus frequency at several $T$. (b) Same away from the FQCP, for $b=2$. Now the transition is continuous. (c) The magnitude of the jump of $\Delta(T, i \pi T)$ at the instability temperature versus $b$. The line is a guide to the eye. (d) $\Delta(T, i \pi T))$ at the lowest $T$ versus $b$.

sition temperature lies between $T_{c}^{l}$ and $T_{c}^{n l}$ and hence remains finite at $b=0$. For $0.9 \lesssim b<b_{c}$, the jump in $\Delta$ at $T_{c}^{n l}$, and consequently the difference between $T_{c}^{l}$ and $T_{c}^{n l}$ is small but finite (see Fig. 11c). The insert shows the reduction of $T_{c}$ at large $b$ due to the decrease in the effective coupling.

The functional form of $T_{c}^{l}(b)$ obtained above agrees qualitatively with that found earlier by Monthoux and Lonzarich 迎. Their result for the maximum $T_{c}^{l}$ is $T_{c, \text { max }}^{l} \sim 0.016 \omega_{0} b^{3 / 2}$. Substituting $b \sim 0.8$, where $T_{c}^{l}$ is maximal in our case, we obtain $T_{c, \text { max }}^{l} \sim 0.011 \omega_{0}$, consistent with our result $T_{c, \max }^{l} \sim 0.013 \omega_{0}$.

Finally, the validity of the Eliashberg treatment requires that three conditions be satisfied. First, typical bosonic momenta $q_{B}$ should be much larger than typical fermionic $\left|k-k_{F}\right|$, i.e., bosons should be slow modes com- 


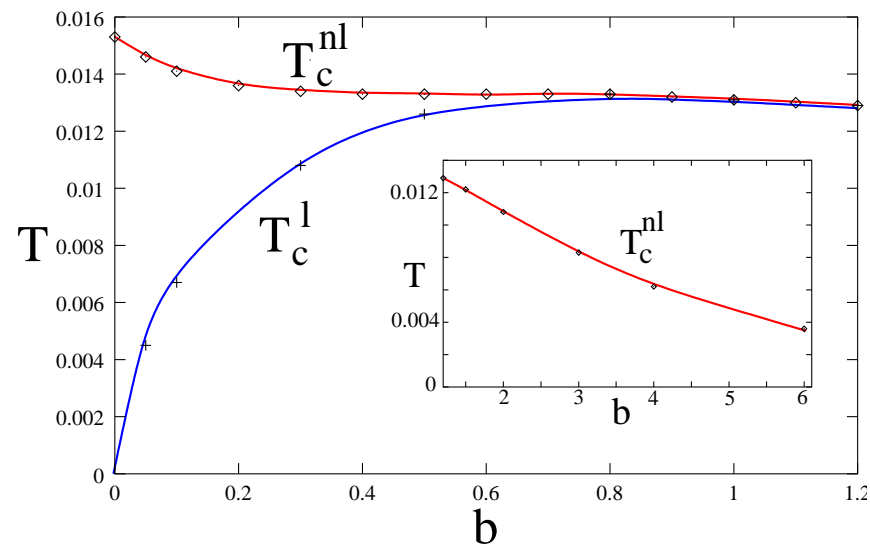

FIG. 2: The phase diagram near the FQCP. In the near vicinity of the FQCP, the transition is of first order, away from the FQCP, to the right of $b_{c}$, it is of second order. For the first order transition, $T_{c}^{n l}$ and $T_{c}^{l}$ are the instability temperatures for the solutions with a large and infinitesimally small gap, respectively. The actual first order transition temperature, $T_{c}$, lies between $T_{c}^{l}$ and $T_{c}^{n l}$. Inset: the reduction of $T_{c}$ at large $b$.

pared to fermions, leading to $\Sigma(k, \omega) \approx \Sigma(\omega)$. A straightforward analysis shows that the typical $q_{B} \sim \alpha k_{F}$, while the typical $\left|k-k_{F}\right| \sim \omega_{0} / v_{F} \sim \alpha^{2} k_{F}$. The Eliashberg theory is therefore valid if $\alpha \ll 1$, i.e., $\omega_{0} \ll E_{F}$. This in turn implies that the physical behavior of the system is universally determined by only low energy excitations. Second, vertex corrections should be small. Generally, this is not possible for typical $q_{B} \ll k_{F}$, as vertex corrections scale with $\xi$. However, for $\alpha \ll 1$, we only require the vertex for $\Omega \ll v_{F} q$ since the typical $v_{F} q_{B}$ well exceeds the typical $\Omega \sim \omega_{0}$. In this limit, vertex corrections are much smaller and only scale as $\log \xi$. They are still non-negligible at the FQCP, where they change the pole in the spin susceptibility into a branch cut [10]. However, we verified that, as in the antiferromagnetic case [9], this only leads to a small renormalization of the prefactors in the gap equation. Third, one should be able to neglect the momentum dependence of the pairing gap, while preserving the gap symmetry, $\Delta\left(\vec{n} k_{F}\right)=-\Delta\left(-\vec{n} k_{F}\right)$. This approximation is again justified by $\alpha \ll 1$, as in this limit, the typical momentum transfers along the Fermi surface $\delta k \sim q_{B} \sim \alpha k_{F}$ are much smaller than $k_{F}$. In this situation, the momentum variation of the gap at typical $\delta k$ only introduces $O(1)$ corrections to the Eliashberg theory [9], which can be safely neglected. Note in passing that the smallness of $\delta k \ll k_{F}$ makes our theory also applicable to real materials (in which a crystalline structure imposes additional constraints on the order parameter symmetry [15]), as it allows one to consider the pairing problem in a local-momentum approximation, ignoring the peculiarities of the gap's momentum dependence.

In summary, we showed that near a FQCP, spin fluctuation exchange gives rise to a strong first order transition into a $p$-wave superconducting state. By choosing a first order transition, the system avoids divergent pairbreaking effects from thermal spin fluctuations. As a result, $T_{c}$ saturates at a nonzero value at criticality. The first order transition persists up to a finite distance from the FQCP, where it becomes second order.

It is our pleasure to thank A. Abanov, D. Khveschenko, D. Pines, A. Tsvelik and Z. Wang for useful discussions. The research was supported by NSF DMR-9979749 (A. Ch.), BSF-1999354 (A.C and A. F) and by DR Project 200153 and the Department of Energy, under contract W-7405-ENG-36. (R.H.) A.C. and D.M. would like to thank Los Alamos National Laboratory for its hospitality during the completion of this project.
[1] A. Chubukov, J. Schmalian and D. Pines condmat/0201140.

[2] P.W. Anderson and P. Morel, Phys. Rev 123, 1911 (1961); for a review see e.g., A.J.Legget Rev. Mod. Phys. 47, 331, 1975..

[3] C. Pfleiderer, G. J. McMullan, S. R. Julian, and G. G. Lonzarich, Phys. Rev. B 55, 8330 (1997); S.S. Saxena et al, Nature 406, 587 (2000).

[4] Ph. Monthoux and G.G. Lonzarich, Phys. Rev. B 59, 14598 (1999).

[5] Z. Wang, W. Mao and K. Bedell, Phys. Rev. Lett. 87, 257001 (2001); K.B. Blagoev, J.R. Engelbrecht and K. S. Bedell, Phys. Rev. Lett 82, 133 (1999).

[6] R. Roussev and A. J. Millis, Phys. Rev. B 63, 140504 (2001).

[7] A.A. Abrikosov and L.P. Gor'kov, Sov. Phys. JETP, 12, 1243 (1961).

[8] P. B. Littlewood and C. M. Varma. Phys. Rev. B 46, 405 (1992).

[9] Ar. Abanov, A. V. Chubukov, and A. M. Finkel'stein,
Europhys. Lett., 54 ,488-494 (2001).

[10] A. Abanov, A. V. Chubukov, J. Schmalian, condmat/0107421.

[11] D. Belitz, T. R. Kirkpatrick and T. Vojta, Phys. Rev. B 55, 9452 (1997); A. Chubukov and D. Maslov, in preparation.

[12] G.Y. Chitov and A.J. Millis, Phys. Rev. Lett. 86, $5337(2001)$.

[13] G.M. Eliashberg, Sov. Phys. JETP 11, 696 (1960); D.J. Scalapino in Superconductivity, Vol. 1, p. 449, Ed. R. D. Parks, Dekker Inc. N.Y. 1969; F. Marsiglio and J.P. Carbotte, in 'The Physics of Conventional and Unconventional Superconductors', Eds. K.H. Bennemann and J.B. Ketterson (Springer-Verlag).

[14] B. L. Altshuler, L.B. Ioffe, and A. J. Millis, Phys. Rev. B 52, 5563 (1995).

[15] M.Sigrist and K.Ueda, Rev. Med. Phys. 63, 239, 1991. 